\title{
Clinical Features and Outcome Analysis of Patients Infected with Severe and Critical COVID-19 Associated Pneumonia
}

\section{Yangmei Xiong}

Zhongnan Hospital of Wuhan University

\section{Ying Feng}

Zhongnan Hospital of Wuhan University

Mengwei Li

People's Hospital of Jingshan

\section{Jing Wang}

Zhongnan Hospital of Wuhan University

\section{Xingguo Zhang}

People's Hospital of Xishui

\section{Xiangyang Chen}

The People's Hospital of Tuanfeng

\section{Xin Rao ( $\nabla$ raoxin4169@163.com )}

Zhongnan Hospital of Wuhan University https://orcid.org/0000-0002-6243-8441

\section{Research}

Keywords: COVID-19, Pneumonia, Severe, Critical, Clinical features, Outcome

Posted Date: March 17th, 2020

DOl: https://doi.org/10.21203/rs.3.rs-17395/v1

License: (9) This work is licensed under a Creative Commons Attribution 4.0 International License. Read Full License 


\section{Abstract}

Background Until now, information on the clinical characteristics of severe and critical patients with COVID-19 is extremely limited.The aim of the present study was to analyse the clinical features of these patients and influencing factors of clinical outcome, and explore treatment effects of prone position on COVID-19 patients with severe ARDS. Methods A retrospective analysis was performed on 55 COVID-19 patients in the ICU of Zhongnan Hospital of Wuhan University from January 6 to February 15, 2020 in Wuhan, China. Case data from each patient were collected and related clinical outcomes on day 14 of ICU admission were recorded. The follow-up deadline was February 29, 2020. Results Of the 55 patients included, 35 were male (63.6\%), with an average age of 63.0 (SD 15.2) years, and $80.0 \%$ were patients over 50 years old. The first three symptoms were fever (36 cases, $65.5 \%)$, fatigue (13 cases, $23.6 \%$ ), and cough (11 cases, $20.0 \%$ ). The rate of invasive mechanical ventilation was $52.7 \%$ (29 cases); on the 14th day of ICU admission, 31 patients(56.4\%) were improved, and 19 (34.5\%) were worsened. On the 14th day after entering the ICU, a comparative analysis showed that peripheral blood CD4, CD8, and NK cell counts in deteriorated patients were significantly lower than those in improved patients $(P<0.05)$.

Meanwhile, concentrations of IL-10, IL-4, IL-6 and TNF- $a$ in deteriorated patients were higher than those in improved patients $(\mathrm{P}<0.05)$. Among a total of 27 prone position sessions, the oxygenation index ( $\mathrm{PaO} 2 /$ FiO2 ) of 9 prone position sessions(33.3\%) improved, and the PaCO2 in arterial blood gas analysis of 5 sessions(18.5\%) improved. Conclusion The majority of patients with severe and critical COVID-19 in the ICU were over 50 years old and male. $52.7 \%$ need invasive mechanical ventilation. On the 14 th day of admission, $56.4 \%$ of the patients improved, $34.5 \%$ of the patients deteriorated. The rate of deaths during hospitalization was $21.8 \%$. The worsening of COVID-19 patients might be related to excessive inflammatory and immune responses. In addition, prone ventilation may improve oxygenation in some COVID-19 patients with severe ARDS, but a significant mortality benefit with proning was uncertain.

\section{Background}

In December 2019, multiple unexplained pneumonia cases occurred in Wuhan, China, and laboratory tests later confirmed that the pathogen was a new coronavirus (SARS-CoV-2) with significantly different genetic characteristics than SARS-CoV and MERS-CoV. On February 11, 2020, the World Health Organization officially named SARS-CoV-2 infected pneumonia as COVID-19 (the 2019 novel coronavirus disease). COVID-19 is mainly manifested by fever and respiratory symptoms, and is transmitted through droplets and contact. COVID-19 is extremely contagious. It has quickly spreaded to the entire Hubei province and affected major provinces and cities in China and even whole world since the first patient appeared in December 2019. The latest epidemiological data analysis of 8866 COVID-19 patients in China ${ }^{[1]}$ showed that the basic reproduction number(R0) of COVID-19 was 3.77 , which was equivalent to severe acute respiratory syndrome (SARS, R0 was 2.9 to 3.3), and much higher than Middle East Respiratory Syndrome (MERS). As of 24:00 on March 4, 2020, there were already 80422 confirmed case and 2984 death in China. 
According to the clinical classification criteria in the fifth edition of the new Coronavirus diagnosis and treatment program ${ }^{[2]}$ issued by the National Health Commission of China, most patients with COVID-19 are light or common, and could recover after bed rest and enhanced supportive treatment. Some severe or critical patients might develop organ dysfunction, such as ARDS, septic shock, acute kidney injury, acute myocardial injury and so on, or even die. A study by Wang $D$ et al. ${ }^{[3]}$ showed that about $26 \%$ of hospitalized COVID-19 patients need to enter the ICU for respiratory and organ function supportive care. Therefore, active and effective treatment of severe and critical COVID-19 patients is the key to reduce the mortality of COVID-19. However, patients in most current clinical studies on COVID-19 ${ }^{[3-6]}$ are light or ordinary patients, and the clinical characteristics of severe and critical patients are extremely limited. And no analysis on the factors affecting the clinical outcome of this part of patients are reported. Through analyzing the clinical characteristics of severe and critical COVID-19 patients and the influencing factors of clinical outcome on the 14th day of admission to the ICU, and exploring the treatment effect of prone position on COVID-19 patients with severe ARDS, we hope to provide evidences for early identification and clinical interventions.

\section{Methods}

\subsection{Research design and patients inclusion}

This study was a retrospective single-center case study, and enrolled all patients diagnosed with COVID19 who were admitted to the ICU isolation ward in Zhongnan Hospital of Wuhan University from January 6 to February 15, 2020. The diagnostic criteria of COVID-19 referred to the fifth edition of the new coronavirus diagnosis and treatment program issued by the Chinese National Health Commission ${ }^{[2]}$. SARS-CoV-2 nucleic acid detection in throat swab specimens of enrolled patients was positive by RTPCR. This study was approved by the Ethics Committee of Zhongnan Hospital of Wuhan University (No.2020045). All patients had signed informed consent when they entered the ICU. Each patient was monitored for clinical outcomes (improvement, stalemate, or deterioration) on the 14th day of ICU admission. The follow-up deadline was February 29, 2020.

\subsection{Data collection}

Through the electronic case system, epidemiological, demographic, clinical and radiological characteristics, laboratory tests, treatments, and clinical outcomes of the enrolled patients were reviewed and recorded. If the first symptoms and contact history could not be obtained from the case system, communications with the patient and family members in person or on the phone were needed. The onset date was the date on which the first symptoms appeared. The time from onset to tracheal intubation, onset to ICU, tracheal intubation, and ICU hospital stay were recorded. The peak body temperature was the highest body temperature throughout the course of the disease. While SARS-CoV-2 nucleic acid in throat swabs was tested in every patient, other respiratory viruses, such as respiratory syncytial virus, influenza A virus, influenza B virus, $\mathrm{H} 7$ avian influenza virus and so on, were also analyzed. Save sputum 
samples for bacterial culture or fungal testing. The recorded laboratory tests included blood routine tests, procalcitonin, liver function, kidney function, electrolytes, coagulation function, and respiratory pathogenic tests, all of which were the results of the first blood draw after ICU admission. Blood samples should be taken before glucocorticoids were used. Prone position therapy was performed for all patients whose $\mathrm{PaO}_{2} / \mathrm{FiO}_{2}$ was still less than $100 \mathrm{mmHg}$ after invasive mechanical ventilation. The prone position duration and blood gas analysis results within 4-6 hours before and after each prone position session were recorded. All data was reviewed by two physicians (Ying Feng and Mengwei Li).

The clinical classification criteria for the severity of COVID-19 patients referred to the fifth edition of the new coronavirus diagnosis and treatment program issued by the Chinese National Health Commission ${ }^{[2]}$. The clinical outcome on the 14th day after entering the ICU referred to improvement, stalemate, ordeterioration. The improvement of condition was defined as the clinical classification changed from severe type when entering the ICU to ordinary type, or from critical to severe or common type; stalemate was defined as no change in clinical classification when compared with which when entering ICU; deterioration was defined as clinical classification from critical type to death, or severe type to critical type or death. Organ damage of every patient was recorded, including ARDS, septic shock, acute kidney injury, and acute myocardial injury. ARDS diagnosis and classification referred to the Berlin definition ${ }^{[7]}$. The diagnosis of septic shock was based on the "2016 Sepsis Rescue Campaign" guidelines ${ }^{[8]}$. The diagnosis of acute kidney injury was based on the Guidelines for Kidney Disease『lmproving Global Outcomes (KDIGO) in 2012 ${ }^{[9]}$. Acute myocardial injury was defined as the blood concentrations of myocardial injury markers, such as high-sensitivity troponin I, exceeding the 99th percentile reference

upper limit or new abnormalities of ECG and echocardiography were displayed ${ }^{[4]}$. Referring to the study of Sahoo JN et al. ${ }^{[10]}$, the improvement of $\mathrm{PaO}_{2} / \mathrm{FiO}_{2}$ after prone postion treatment was defined as at least $20 \%$ increase of $\mathrm{PaO}_{2} / \mathrm{FiO}_{2}$ compared with which before prone position; $\mathrm{PaCO}_{2}$ improvement was defined as at least $6 \mathrm{mmHg}$ decrease of $\mathrm{PaCO}_{2}$ in arterial blood after prone postion compared with which before prone position.

\subsection{SARS-CoV-2 nucleic acid detection}

Real-time reverse transcription polymerase chain reaction (RT-PCR) was used to determine SARS-CoV-2 RNA in throat swab specimens. RT-PCR detectedopen reading frame $1 \mathrm{ab}$ (ORF1ab) and nucleocapsid protein $(\mathrm{N})$ target genes at the same time. Target gene 1 (ORF1ab): forward primer CCTGGTGGGTTTTACACTTAA; reverse primer ACGATTGTGCATCAGCTGA; probe 5'-VICCCGTCTGCGGTATGTGGAAAGGTTATGG-BHQ1-3 '. Target gene 2 (N): forward primer GGGGAACTTCTCCTGCTAGAAT; reverse primer CAGACATTTTGCTCTCAAGCTG; and probe 5'-FAMTTGCTGCTGCTTGACAGATT-TAMRA-3'. TheRT-PCR was performed as follows: incubation at $50{ }^{\circ} \mathrm{C}$ for 15 minutes and $95^{\circ} \mathrm{C}$ for 5 minutes, denaturation at $94{ }^{\circ} \mathrm{C}$ for 40 cycles for 15 seconds, extension at 55 ${ }^{\circ} \mathrm{C}$ and collection of fluorescence signal for 45 s. SARS-CoV-2 nucleic acid detection kit was issued by 
China National Center for Disease Control and Prevention, and the manufacturer was Shanghai Biological Bacterial Medical Technology Co Ltd.

\subsection{The role of funding sources}

The fund sponsor did not participate in the project design, data collection, data analysis, and thesis writing of this research. To guarantee the accuracy and integrity of the research, corresponding author was responsible for quality control and review. The final version of the submission was determined by all authors.

\subsection{Data analysis}

Data analysis was performed using SPSS 20.0 statistical software. Measurement data that was normally distributed was expressed as mean(standard deviation) $[X(S D)]]$, and comparison between groups was performed using independent sample $t$ test. Measurement data that was not normally distributed was expressed as median (interquartile range) $[M(I Q R)]]$, and group comparisons were made using MannWhitney U test. Count data was expressed in $N(\%)_{\text {," }}$ and comparisons between groups were performed using the $X^{2}$ test or Fisher's exact probability method. The difference was statistically significant at $P$ $<0.05$.

\section{Results}

\subsection{Demographic and epidemiological characteristics of patients}

Among the 55 patients enrolled, 35 (63.6\%)were male and 20 (36.4\%) were female. The age ranged from 29 to 92 years old, with an average age of 63.0 (15.2). The majority of patients were over 50 years old, accounting for $80.0 \%$. Some patients $(56.4 \%)$ had normal body mass index, and $40.0 \%$ were overweight or obese; in the aspect of occupation, 34 patients $(61.8 \%)$ were retirees, 3 patients (5.5\%) were medical staff; 26 patients $(47.3 \%)$ had hypertension, 12 patients $(21.8 \%)$ had diabetes, and 14 patients $(25.5 \%)$ had no any underlying disease. In terms of contact history, 2 patients had visited the South China Seafood Market before their onset, and 4 patients visited Vegetable market (not South China Seafood Market) near residence, 7 patients had a clear history of exposure to COVID-19 patients. Details show in Table 1.

\subsection{Clinical characteristics of patients}

The first three symptoms of patients enrolled were fever (36 cases, $65.5 \%)$, fatigue ( 13 cases, $23.6 \%$ ), and cough (11 cases, 20.0\%). One case had diarrhea as the first symptom and 11 cases (20.0\%) had 
gastrointestinal symptoms such as diarrhea, nausea and vomiting during the onset of disease; 43 patients $\left(78.2 \%\right.$ ) had a peak body temperature of more than $38.5^{\circ} \mathrm{C}$ during the course of the disease, and 1 elderly woman had no fever during the course of the disease. The scores of SOFA and APACHEII were 5 (4-7) and 17 (11-27), respectively. The proportion of patients with invasive mechanical ventilation was $52.7 \%$ ( 29 cases); 8 patients (14.5\%) were treated with ECMO(extracorporeal membrane oxygenation).

The average time was 11.3 (5.4) days from onset to tracheal intubation, the average time to ICU admission was 10.5 (4.5) days; tracheal intubation time was 6 (3-10) days, and ICU hospitalization time was $8(5-13)$ days. On the 14th day after admission to the ICU, 31 patients $(56.4 \%)$ were improved, 5 patients(9.1\%) were stalemated, and 19 (34.5\%) were deteriorated. As of the end of the follow-up time, 36 patients(65.4\%)discharged from ICU, 12 patients(21.8\%)died and 7(12.7\%) were still hospitalized in the ICU. Show in Table 2.

\subsection{Laboratory Parameters of patients}

Among the patients enrolled, 8 cases (14.5\%) were positive for influenza A virus in throat swabs, 7 $(12.7 \%)$ were positive for influenza B virus, and 50 cases $(90.9 \%)$ were negative for common respiratory pathogens, such as Mycoplasma pneumoniae, Chlamydia pneumoniae, Respiratory syncytial virus and so on. Blood tests of 18 patients (32.7\%) showed low white blood cell counts, 25 patients (45.5\%) had normal white blood cell counts, and 12 patients $(21.8 \%)$ had white blood cell counts above the normal range. The absolute counts of lymphocytes in most patients $(48,87.3 \%)$ were under the normal range; 18 patients(32.7\%) had normal procalcitonin, and 29 patients(52.7\%) had mildly elevated procalcitonin (< $1.00 \mathrm{ng} / \mathrm{ml}) .11$ patients $(20.0 \%)$ had acute kidney injury, $25(45.5 \%)$ had septic shock, $11(20.0 \%)$ had acute myocardial injury. Secondary infection occurred in 19 patients(34.5\%) during hospitalization. 10 patients (18.2\%) had sputum cultured as Acinetobacter baumannii complex and 7 (12.7\%) had sputum cultured as Candida. When patients were admitted to the ICU, the ARDS classification of patients was mild in 16 cases (29.1\%), 24 cases of moderate (43.6\%), 15 cases of severe (27.3\%). Show in Table 3.

\subsection{Comparison of clinical characteristics of patients with improvement and deterioration on the 14th day after ICU admission}

On the 14th day after ICU admission, the clinical characteristics of patients who improved and deteriorated indicated that the average age of deteriorated patients was older $(P<0.05)$, and SOFA(Sequential organ failure assessment) and APACHEII(Acute physiology and chronic health evaluation II) scores of deteriorated patients were higher $(\mathrm{P}<0.05)$. Analysis of Lymphocyte subsets showed that $C D 4, C D 8$, and NK cell counts in peripheral blood were significantly lower in deteriorated patients $(P<0.05)$. Meanwhile, the concentrations of IFN- $y, I L-10, I L-4, I L-6$ and TNF- $\alpha$ in peripheral blood of deteriorated patients were higher than those of patients with improved disease $(P<0.05)$. In 
addition, patients with worsening condition were more likely to develop acute kidney injury, septic shock, acute myocardial injury, and secondary infections during the disease course $(P<0.05)$. Show in Table 4.

\subsection{Changes of oxygenation index in patients before and after prone position ventilation}

Among the patients enrolled, 13 patients were ventilated in the prone position, 3 patients were in the prone position once, 6 were in the prone position twice, and 4 were in the prone position three times; the average oxygenation index before the first prone position was 81.0 (16.4) $\mathrm{mmHg}$; total sessions of prone position were 27, each prone position session was $15(13-16)$ hours. During the treatments, oxygenation index was improved after 9 sessions $\left(\mathrm{PaO}_{2} / \mathrm{FiO}_{2}\right.$ increased at least $20 \%$ after prone position compared with that before prone position), arterial blood $\mathrm{PaCO}_{2}$ was improved after 5 sessions (arterial blood $\mathrm{PaCO}_{2}$ decreased at least $6 \mathrm{mmHg}$ after prone position). Show in Table 5.

\section{Discussion}

Until now, it is the first clinical study to specifically analyze the clinical characteristics, clinical outcomes, and prone position treatment effects of severe and critical patients with COVID-19. Of the 55 patients enrolled, $80.0 \%$ were over 50 years old, and most patients had no clear history of COVID-19 exposure before onset. Common first symptoms were fever, fatigue, and cough. $1.8 \%$ of patients developed diarrhea which was the atypical symptom at onset of illness, and $20.0 \%$ of patients experienced gastrointestinal symptoms such as diarrhea, nausea and vomiting during the course of the disease, suggesting the possibility of fecal-oral transmission for SARS-CoV-2. When most patients entered the $\mathrm{ICU}$, the infection index was not high, but the lymphocytes were low. During the development of the disease, common organ damage was acute kidney injury, septic shock, and acute myocardial injury. Common secondary infections were Acinetobacter baumannii complex and Candida. In addition, almost half of the patients required invasive mechanical ventilation, and $14.5 \%$ of patients received ECMO. On the 14th day after ICU admission, the condition of 31 patients(56.4\%) improved and the condition of 19 patients(34.5\%) deteriorated. As of February 29, 2020, 65.4\% had discharged from ICU, 12 patients(21.8\%) died, and $12.7 \%$ were still hospitalized in the ICU.

Similar to SARS-CoV and MERS-CoV infections, there are also gender differences in susceptibility to SARS-CoV-2. An analysis of clinical data of 99 patients with COVID-19 indicated that number of male patients were approximately twice than female patients ${ }^{[5]}$. Among severe and critical patients in ICU, it was similar that men were far more than women. This gender-related difference in susceptibility may result from the secretion of female estrogen and the expression of immune response genes on the $X$ chromosome ${ }^{[11]}$. Studies have confirmed that estrogen has important immunomodulatory effects, which plays an immunosuppressive role at high concentrations and an immune activation role at low concentrations $^{[12,13]}$. However, it is worth noting that there was no significant difference in clinical 
outcomes between male and female patients on the 14th day of ICU admission (worsened patients: male $15 / 35 \mathrm{VS}$ female $4 / 20, P=0.086$ ). This might be related to inflammatory storm in the later development of disease, which accompanied serious imbalance of the body's immune response, resulting in no obvious gender difference.

At present, pathological mechanism of SARS-CoV-2 damage to human body is not clear. It was suggested that, similar to SARS and MERS, inflammatory response and immune damage played important roles in the occurrence and development of COVID-19. Analysis of laboratory results of 41 patients with COVID-19 by Huang $C$ et al. showed that plasma concentrations of IL2, IL7, IL10, GSCF, IP10, MCP1, MIP1A and TNF-a were significantly higher in ICU patients than that in non-ICU cases ${ }^{[4]}$. On January 27, 2020, Wang Fusheng, a academician in the Fifth Medical Center of the General Hospital of the Chinese People's Liberation Army, performed pathological examination of a dead patient with COVID19 through postmortem biopsies. He found lymphocyte-dominated mononuclear cells inflammatory infiltration in the interstitial tissue of both lungs of the patient. Meanwhile, flow cytometry analysis of peripheral blood indicated that number of $\mathrm{CD} 4^{+}$and $\mathrm{CD} 8^{+}$cells was reduced remarkably. However, both were in over-activated statement. And among $\mathrm{CD} 4^{+}$cells, CCR4 ${ }^{+}, \mathrm{CCR}^{+}$, and Th17 subsets with high proinflammatory effects increased ${ }^{[14]}$. Similar results were obtained in our study. A comparative analysis of lymphocyte subsets in patients on the 14th day of ICU showed that the absolute counts of $\mathrm{CD} 4^{+}, \mathrm{CD} 8^{+}$, and NK cells in peripheral blood of improved patient were significantly higher than that in worsend patients. At the same time, pro-inflammatory cytokines, such as IFN- - , IL-6, TNF-a, and anti-inflammatory cytokines, such as IL-10, IL-4, in deteriorated patients were higher than those in improved patients. This result suggests that inflammatory storm may play an important role in deterioration of COVID-19 patients. It is envisaged that glucocorticoids, a commonly used immunosuppressive drugs in the clinic, may have certain effects on COVID-19. However, some experts proposed that while inhibiting lung inflammation, glucocorticoids could also suppress immune response and pathogen clearance ${ }^{[15]}$. At present, the treatment of glucocorticoids on COVID-19 is still controversial. It still need large-scale randomized clinical trials to confirm whether glucocorticoids can accurately improve the clinical outcome.

There is no specific treatment strategy for COVID-19. The main treatment is respiratory support. As a treatment strategy recommended in the guidelines for mechanical ventilation in patients with ARDS ${ }^{[16]}$, prone position ventilation is also considered in COVID-19 patients with severe ARDS. Theoretically, prone position ventilation could improve oxygenation of patients by reducing gravity-dependent atelectasis, lowering pleural pressure gradients, and improving ventilation-perfusion ratios. However, its effect on mortality in ARDS patients is uncertain. The latest meta-analysis on prone ventilation, publishing in the annual report of the American Thoracic Society in 2017, enrolled 8 randomized controlled clinical trials (2129 patients). It was indicated that no significant difference of 28-day mortality was observed between ARDS patients with prone ventilation and supine ventilation. And subgroup analysis suggested that prolonged prone ventilation duration greater than $12 \mathrm{~h} / \mathrm{d}$ might be beneficial in reduced mortality in patients with severe ARDS ${ }^{[17]}$. To date, no related researches on the effectiveness of prone ventilation in 
patients with COVID-19 and ARDS were reported. In this study, 13 patients underwent prone ventilation during treatment. Data analysis showed that $\mathrm{PaO}_{2} / \mathrm{FiO}_{2}$ improved $\left(\mathrm{PaO}_{2} / \mathrm{FiO}_{2}\right.$ increased at least $20 \%$ after prone position) in $33.3 \%$ of all prone position sessions. Arterial blood gas analysis showed that $\mathrm{PaCO}_{2}$ improved (the arterial blood $\mathrm{PaCO} 2$ decreased at least $6 \mathrm{mmHg}$ after prone position) in $18.5 \%$ of prone sessions. Of these 13 patients, 8 patients died, 4 patients were still hospitalized in the ICU, and only 1 patient discharged from ICU after his condition improved. Therefore, we speculated that prone ventilation might improve the oxygenation of some COVID-19 patients with severe ARDS, but a significant mortality benefit with proning was uncertain. However, as the number of patients in this study is relatively small, our speculation need to be confirmed through further studies with large samples.

There are some limitations in this study. Firstly, the number of cases is relatively small, so it is impossible to use multivariate analysis of variance to analyze the risk factors for the worsening of the disease on the 14th day of ICU admission. It will be more representative to use large sample data to analyze the clinical characteristics of severe and critical COVID-19 patients. Secondly, as COVID-19 patients need to return to the general isolation ward for further treatment after being discharged from ICU, the follow-up period in this study is relatively short, and the final clinical outcome (death or cure) of the patient cannot be fully calculated. Two patients in this study returned to the general isolation ward for continue treatment after their condition improved, but suddenly respiration worsening happened and eventually patients died. Therefore, the analysis of the final clinical outcome may be more instructive for the treatment of COVID19 patient. Finally, recent research ${ }^{[18]}$ found that SARS-CoV-2 load in respiratory specimens of COVID19 patients was closely related to the severity of the disease and the degree of lung injury. It was believed that early and continuous monitoring of virus load may have certain predictive effects on disease development. However, due to lack of data, this study failed to analyze the correlation between SARSCoV-2 load in respiratory specimens from COVID-19 patients and clinical outcome.

\section{Conclusion}

The majority of patients with severe and critical COVID-19 who need to stay in the ICU were over 50 years old and male. $52.7 \%$ need invasive mechanical ventilation. On the 14 th day of admission, $56.4 \%$ of the patients improved, $34.5 \%$ of the patients deteriorated. The rate of deaths during hospitalization was $21.8 \%$. The worsening of patients with COVID-19 might be related to excessive inflammatory and immune responses. In addition, prone ventilation may improve oxygenation in some COVID-19 patients with severe ARDS, but a significant mortality benefit with proning was uncertain.

\section{Declarations}

\section{Abbreviations}

COVID-19: 2019 novel coronavirus disease; ICU: Intensive care unit; SARS: Severe acute respiratory syndrome; MERS: Middle east respiratory syndrome; KDIGO: Kidney disease冈improving global outcomes; ARDS: Acute respiratory distress syndrome; RT-PCR: Real-time reverse transcription polymerase chain 
reaction; SD: standard deviation; IQR: Interquartile range; SOFA: Sequential organ failure assessment; APACHEIl: Acute physiology and chronic health evaluation II; ECMO: Extracorporeal membrane oxygenation

\section{Authors' contributions}

YMX designed the study, analyzed data, and contributed to writing this manuscript. YF designed the study, collected and analyzed data. MWL, JW, XGZ, and XYC helped with data collection and analyzation. XR designed and supervised the study and drafted the manuscript. All authors have read and approved the final manuscript.

\section{Funding}

This work was supported by grants from Research Foundation of Health and Family Planning Commission of Hubei Province (No. WJ2017M041).

\section{Availability of data and materials}

The datasets used in the present study are available from the first authors and corresponding author on reasonable request.

\section{Ethics approval and consent to participate}

This study was approved by the Ethics Committee of Zhongnan Hospital of Wuhan University (No.2020045). All patients had signed informed consent when they entered the ICU.

\section{Consent for publication}

Not applicable.

\section{Competing interests}

The authors declare that they have no competing interests.

\section{References}

[1]Yang Y, Lu Q, Liu M, et al. Epidemiological and clinical features of the 2019 novel coronavirus outbreak in China[J]. medRxiv, 2020:2020-2022. 
[2]National Health Commission of China:The new coronavirus diagnosis and treatment program(the fifth edition)[J]. 2020. http://www.nhc.gov.cn.Accessed 5 March 2020.

[3]Wang D, Hu B, Hu C, et al. Clinical characteristics of 138 hospitalized patients with 2019 novel coronavirus-infected pneumonia in Wuhan, China[J]. JAMA, 2020:10-1001.

[4]Huang C, Wang Y, Li X, et al. Clinical features of patients infected with 2019 novel coronavirus in Wuhan, China[J]. Lancet (London, England), 2020,395(10223):497-506.

[5]Chen N, Zhou M, Dong X, et al. Epidemiological and clinical characteristics of 99 cases of 2019 novel coronavirus pneumonia in Wuhan, China: a descriptive study[J]. Lancet (London, England), 2020,395(10223):507-513.

[6]Li Q, Guan X, Wu P, et al. Early transmission dynamics in Wuhan, China, of novel coronavirus-infected pneumonia[J]. The New England journal of medicine, 2020:10-1056.

[7]Ranieri VM, Rubenfeld GD, Thompson BT,et al. Acute respiratory distress syndrome:the Berlin Definition[J]. JAMA, 2012,307(23):2526-2533.

[8]Rhodes A, Evans L E, Alhazzani W, et al. Surviving sepsis campaign: International guidelines for management of sepsis and septic shock: 2016[J]. Intensive care medicine, 2017,43(3):304-377.

[9]Kellum JA, Lameire N, KDIGO A G W G. Diagnosis, evaluation, and management of acute kidney injury: a KDIGO summary (Part 1)[J]. Critical care (London, England), 2013,17(1):204.

[10]Sahoo JN, Gurjar M, Mohanty K, et al. Prone ventilation in H1N1 virus-associated severe acute respiratory distress syndrome: A case series[J]. International journal of critical illness and injury science, 2019,9(4):182-186.

[11]Channappanavar R, Fett C, Mack M, et al. Sex-based differences in susceptibility to severe acute respiratory syndrome coronavirus infection[J]. Journal of immunology (Baltimore, Md.: 1950), 2017,198(10):4046-4053.

[12]Klein SL, Flanagan KL. Sex differences in immune responses[J]. Nature reviews. Immunology, 2016,16(10):626-638.

[13]Roved J, Westerdahl H, Hasselquist D. Sex differences in immune responses: Hormonal effects, antagonistic selection, and evolutionary consequences[J]. Hormones and behavior, 2017,88:95-105.

[14]Xu Z, Shi L, Wang Y, et al. Pathological findings of COVID-19 associated with acute respiratory distress syndrome[J]. The Lancet. Respiratory medicine, 2020:S2213-S2600.

[15]Russell CD, Millar JE, Baillie JK. Clinical evidence does not support corticosteroid treatment for 2019nCoV lung injury[J]. The Lancet, 2020,395(10223):473-475. 
[16]Chinese Thoracic Society: Guidelines for mechanical ventilation in patients with ARDS[J]. National Medical Journal of China, 2016,96(6):404-424.

[17]Munshi L, Del Sorbo L, Adhikari NK J, et al. Prone position for acute respiratory distress syndrome. a systematic review and meta-analysis[J]. Annals of the American Thoracic Society, 2017,14(Supplement_4):S280-S288.

[18]Liu Y, Yang Y, Zhang C, et al. Clinical and biochemical indexes from 2019-nCoV infected patients linked to viral loads and lung injury[J]. Science China. Life sciences, 2020:10-1007.

\section{Tables}

Due to technical limitations, Tables 1 - 5 are only available for download from the Supplementary Files section.

\section{Figures}


Retrospectively enrolled COVID-19 patients who were admitted to the ICU isolation ward from January 6 to February 15, 2020

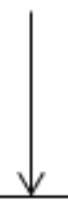

Recorded epidemiological, demographic, clinical and radiological characteristics, laboratory tests, treatments, and clinical outcomes of the enrolled patients

Analysed the clinical features of these patients and influencing factors of clinical outcome, and explored treatment effects of prone position on COVID-19 patients with severe ARDS

\section{Figure 1}

Flow chart of the study.

\section{Supplementary Files}

This is a list of supplementary files associated with this preprint. Click to download.

- Tables.docx 